\title{
About the International Development Research Centre
}

To achieve self-reliance, poor communities need answers to questions like: How can we grow more and healthier food? Protect our health? Create jobs? IDRC, Canada's International Development Research Centre, supports research in developing countries to answer these questions. IDRC also encourages sharing this knowledge with policymakers, other researchers, and communities around the world. The result is innovative, lasting local solutions that aim to bring choice and change to those who need it most. 